\title{
Conservação pós-colheita de fissális e desempenho produtivo em condições edafoclimáticas de Minas Gerais
}

\author{
Daniel Fernandes da Silval, Fabíla Villa², Fabiane Karine Barp ${ }^{3}$, Maria Cristina Copello Rotili ${ }^{4}$ \\ Diego Ricardo Stumm ${ }^{3}$
}

\begin{abstract}
RESUMO
O Physalis sp., popularmente conhecido como fisális, é um gênero com mais de 100 espécies, pertencente à família Solanaceae, caracterizado pelo cálice concrescido, que envolve e protege o fruto contra herbívoros e intempéries. Os frutos, de sabor agridoce, são muito utilizados na culinária, podendo também ser processados em geleias, doces e licores, consumidos in natura, por seu alto teor de vitamina $\mathrm{C}$ e antioxidantes, além de apresentarem inúmeras propriedades medicinais. Este trabalho objetivou analisar a produtividade de fisális na região sul do Estado de Minas Gerais e observar aspectos de sua conservação pós-colheita. Os experimentos relacionados com a produtividade foram instalados na Fazenda Experimental da EPAMIG, em Maria da Fé, MG, e os demais experimentos, relacionados com a conservação pós-colheita, instalados no Laboratório de Tecnologia de Alimentos da UNIOESTE. Para avaliação da produtividade, instalou-se experimento contendo três blocos, com quatro plantas, e, para avaliação pós-colheita, foram utilizadas três repetições de cinco frutos, armazenados em câmara controlada tipo B.O.D, sem atmosfera modificada, por 28 dias, à temperatura de $5^{\circ} \mathrm{C}$, e avaliados no momento da instalação, aos 7, 14, 21 e 28 dias. Foram avaliados diâmetros longitudinal e transversal $(\mathrm{mm})$, peso médio do fruto (g), com e sem cálice, peso total da colheita $(\mathrm{g})$ e teor de sólidos solúveis totais. Em laboratório, avaliaram-se perda de peso, acidez (ATT), pH, sólidos solúveis totais (SST) e teor de vitamina C. Os frutos cultivados em Minas Gerais apresentaram características semelhantes às de frutos produzidos em regiões tradicionais de cultivo e podem facilmente ser armazenados por um período de 28 dias.
\end{abstract}

Palavras-chave: Physalis peruviana L., pós-colheita, pequenas frutas, armazenamento de frutos.

\section{ABSTRACT}

\section{Postharvest and fruit production of cape gooseberry in Minas Gerais State, Brazil}

Physalis sp., is a genus of plant popularly known as cape gooseberry. The enlarged calyx that surrounds and protects the fruit against herbivores and weather characterizes the genus, which contains over than one hundred species belonging to the Solanaceae family. The bittersweet fruits are widely used in cooking, and can also be processed into jellies, jams and liqueurs, or consumed raw. The fruits present high content of vitamin $\mathrm{C}$ and antioxidants, and therefore, contain numerous medicinal properties. This study aimed to evaluate the productivity of cape gooseberry in southern Minas Gerais State, and to observe the aspects of its postharvest. The experiments related to the productivity were installed at the Experimental Farm of EPAMIG, in Maria da Fé, Minas Gerais State, Brazil, while other experiments on postharvest were carried out in the Laboratory of Food Technology of UNIOESTE. To evaluate the productivity, the

Recebido para publicação em 29/12/2011 e aprovado em 08/07/2013

'Graduando em Ciências Biológicas. Centro de Ciências Biológicas e da Saúde, Universidade Estadual do Oeste do Paraná, Campus Cascavel, Rua Universitária, 2069, Bairro Jardim Universitário, 85819-110, Cascavel, Paraná, Brasil. daniel_eafi@yahoo.com.br (autor para correspondência).

${ }^{2}$ Engenheira-Agrônoma, Doutora. Centro de Ciências Agrárias, Universidade Estadual do Oeste do Paraná, Campus Marechal Cândido Rondon, Rua Pernambuco 1777, Centro, 85.960-000, Marechal Cândido Rondon, Paraná, Brasil. fvilla2003@hotmail.com

${ }^{3}$ Graduandos em Agronomia. Centro de Ciências Agrárias, Universidade Estadual do Oeste do Paraná, Campus Marechal Cândido Rondon, Rua Pernambuco 1777, Centro, 85.960000, Marechal Cândido Rondon, Paraná, Brasil. fabybarp@ hotmail.com; diego_stumm@hotmail.com

${ }^{4}$ Nutricionista. Centro de Ciências Agrárias, Universidade Estadual do Oeste do Paraná, Campus Marechal Cândido Rondon, Rua Pernambuco 1777, Centro, 85.960-000, Marechal Cândido Rondon, Paraná, Brasil. mcrotili@ hotmail.com 
experiment was settled containing three blocks with four plants, and to evaluate the postharvest behavior, were used three replicates of five fruits, stored in a controlled temperature chamber BOD, without modified atmosphere, for 28 days at $5^{\circ} \mathrm{C}$. The evaluations were performed at install time, and then after 7, 14, 21 and 28 days of storage. We assessed longitudinal and transverse diameter $(\mathrm{mm})$, average fruit weight $(\mathrm{g})$ with and without calyx, the total harvest weight (g) and total soluble solids. In the laboratory we assessed weight loss, acidity (TTA), pH, total soluble solids (TSS) and vitamin C content. The fruits produced in Minas Gerais had similar characteristics compared to those produced in traditional areas of cultivation and can easily be stored for a period of 28 days.

Key words: Physalis peruviana L., postharvest fruit, berries, fruit conservation.

\section{INTRODUÇÃO}

O Physalis sp., popularmente conhecido como fisális, é um gênero com mais de 100 espécies, pertencente à família Solanaceae, que se caracteriza pelo cálice concrescido, que envolve e protege o fruto contra herbívoros e intempéries. Dentre as muitas espécies do gênero, algumas, como Physalis peruviana L., apresentam potencial de exploração agronômica, por seus compostos nutricionais e medicinais, relatados, há tempos, na cultura popular e, mais recentemente, por pesquisadores de inúmeras instituições (Lima 2009a).

A Physalis peruviana é uma planta de porte herbáceo, tendo, em média, um metro de altura, preferencialmente de ambientes sombreados ou de meia luz, que apresenta caule muito piloso e flores amarelas, pentâmeras, inteiramente soldadas, com mancha roxa em cada uma de suas pétalas. $\mathrm{O}$ fruto é carnoso, esférico ou ovoide, medindo cerca de $2 \mathrm{~cm}$ de diâmetro, fortemente odorífero, belo por sua coloração amarelo-alaranjada quando maduro, apresentando numerosas sementes pequenas (Castañeda, 1961).

Cultivado pela primeira vez no Cabo da Boa Esperança, o fisális espalhou-se pelo mundo como planta ornamental (Rufato et al., 2008). Atualmente é cultivado em diversos países do hemisfério Sul, principalmente Colômbia e África do Sul, maiores produtores mundiais da fruta (Lima et al., 2009b).

No Brasil, essa fruta ainda é consumida como exótica, com preço bastante elevado, em função do alto valor agregado, em decorrência da produção limitada, do difícil manejo da colheita, da alta exigência em mão de obra, dos cuidados no transporte e na armazenagem e por serem seus frutos altamente perecíveis, além da escassez de informação a respeito do cultivo dessa espécie (Pereira, 2007).

Buscando esclarecer alguns aspectos sobre o cultivo de fisális, objetivou-se, com este trabalho, avaliar a produtividade e alguns caracteres relacionados com a póscolheita de frutos, cultivados na região de Maria da Fé, alto da Serra da Mantiqueira, sul de Minas Gerais.

\section{MATERIAL E MÉTODOS}

O experimento foi instalado na Fazenda Experimental da Empresa de Pesquisa Agropecuária de Minas Gerais (EPAMIG), localizada no município de Maria da Fé, sul de Minas Gerais. As coordenadas da Fazenda são 22 $18^{\prime}$ '54" S e $45^{\circ} 22^{\prime} 26^{\prime \prime} \mathrm{O}$, com classificação climática, segundo Köppen, tipo Cwb, clima temperado chuvoso (mesotérmico), também denominado subtropical de altitude. $\mathrm{O}$ município caracteriza-se por apresentar temperatura média anual de $17^{\circ} \mathrm{C}$, temperatura média máxima de $23,3^{\circ} \mathrm{C}$, mínima média de $10,1^{\circ} \mathrm{C}$, precipitação anual de $1.738,6 \mathrm{~mm}$ e altitude média de $1.276 \mathrm{~m}$; com predomínio de relevo ondulado (ALMG, 2005).

As mudas de fisális foram obtidas a partir de sementes oriundas da Universidade Federal de Pelotas (UFPel). As sementes foram semeadas em maio de 2010, em bandejas de tubetes, com 96 células, contendo substrato composto por terra de subsolo, matéria orgânica e areia $(7: 2: 1, \mathrm{v} /$ $\mathrm{v} / \mathrm{v})$ e mantidas em telado com $30 \%$ de sombreamento, irrigadas duas vezes ao dia, e adubadas conforme indicação para cultura do tomateiro. As plantas foram cultivadas até início de setembro, quando atingiram tamanho médio de 10 a $15 \mathrm{~cm}$, ideal para o transplantio.

$\mathrm{O}$ transplantio foi realizado para área previamente preparada, em covas de $40 \times 40 \times 40 \mathrm{~cm}$, adubadas com matéria orgânica e adubo químico, conforme análise de solo, em um espaçamento de $1,5 \times 3 \mathrm{~m}$. O delineamento experimental foi totalmente casualizado, com três blocos de quatro plantas. Os tratos culturais foram realizados conforme indicação para outras solanáceas, como berinjela, jiló e pimentão. O controle de pragas e doenças foi realizado, conforme ocorrência específica, com inseticidas; sem irrigação, tendo em vista a rusticidade das plantas e a boa distribuição pluviométrica nas épocas mais críticas.

Aos cinquenta dias, em média, após o transplantio, as plantas emitiram o primeiro botão floral e deram início ao processo reprodutivo, permitindo a primeira colheita dos frutos completamente maduros, bem amarelados e com o cálice completamente seco, em janeiro de 2011. Desde

Rev. Ceres, Viçosa, v. 60, n.6, p. 826-832, nov/dez, 2013 
então, colheitas e avaliações físicas dos frutos passaram a ser feitas a cada três dias, sendo avaliados: diâmetro longitudinal ( $\mathrm{mm})$, diâmetro transversal $(\mathrm{mm})$, peso médio do fruto $(\mathrm{g})$, com e sem cálice, peso total da colheita (g), com e sem cálice, e sólidos solúveis totais.

Para determinação dos parâmetros físicos, foram utilizados dez frutos por bloco, medidos com paquímetro digital e pesados com balança analítica Shimadzu Corporation modelo TX. Esse procedimento ocorreu em todo período produtivo, que se estendeu de janeiro a maio de 2011.

Em meados do período produtivo, no mês de março, amostras foram retiradas e enviadas ao laboratório de Tecnologia de Alimentos da Universidade Estadual do Oeste do Paraná (Unioeste), para complementação das análises físicas e de parâmetros relacionados com a conservação pós-colheita dos frutos, como sólidos solúveis (SS), acidez total titúlavel, pH e relação SS/ATT.

Os frutos foram armazenados em câmara controlada tipo B.O.D., sem atmosfera modificada, por um período de 28 dias, a uma temperatura de $5^{\circ} \mathrm{C}$. Os frutos foram avaliados no momento na instalação do experimento, 7, 14, 21 e 28 dias após a instalação do experimento. O delineamento experimental utilizado foi o de blocos casualizados, com três blocos de cinco frutos.

Foram avaliados a presença de doença, a perda de massa dos frutos, a percentagem de suco dos frutos, os teores de sólidos solúveis (SS), medidos em graus brix, a cor, o pH, a acidez total titulável (ATT), o teor de vitamina $\mathrm{C}$ e a relação $\mathrm{SS} / \mathrm{ATT}$.

Para determinação dos sólidos solúveis totais e da acidez total titulável, aplicou-se metodologia descrita pelo Instituto Adolfo Lutz (IAL, 1985). O pH foi determinado por medição direta com potenciômetro digital de bancada e, para cor e presença de doenças, utilizou-se método visual, estabelecendo escalas de 1 a 6 , em que: 1 corresponde ao menor valor (menos colorido e menor incidência de doença) e 6 ao maior (mais maduros e maior incidência de doenças).

Para determinação da quantidade de vitamina $\mathrm{C}$, foi utilizado o método padrão da AOAC, modificado por Benassi \& Antunes (1988), que substituíram a solução de extração padrão (solução de ácido metafosfórico) por solução de ácido oxálico; por esse método, $5 \mathrm{~mL}$ de amostra foram triturados com $50 \mathrm{~mL}$ de ácido oxálico a $2 \%$, filtrados para remoção das sementes com uso de uma peneira. O conteúdo foi transferido para béquer com volume de $250 \mathrm{~mL}$ e adicionado mais $50 \mathrm{~mL}$ de ácido oxálico. Desse material, coletou-se amostra de $5 \mathrm{~mL}$, para titulação com 2,6-dicloro-fenol-indofenol (DCFI), sendo o ponto de viragem detectado visualmente, quando a solução muda sua coloração de descolorida para rósea.

Os resultados obtidos foram submetidos à análise de variância e teste de Scott-Knott, a 5\% de significância, pelo programa estatístico SISVAR (Ferreira, 2000).

\section{RESULTADOS E DISCUSSÃO}

Os resultados obtidos para teor de sólidos solúveis (SS) foram significativos, podendo-se notar aumento expressivo da concentração de sólidos, a partir do sétimo dia de armazenamento dos frutos de fisális. Quanto ao comportamento da variável ao longo do período de colheita, podem-se observar variações significativas, com resultados superiores para as últimas colheitas, que apresentaram maiores teores médios de SS (Figura 1). Estes valores podem ser atribuídos à diminuição da pluviosidade, com o fim da estação chuvosa na região, no mês de março de 2011, concentrando-se mais o suco celular das células, por causa da indisponibilidade de água para absorção pela planta, o que pode ser observado em trabalhos de Nogueira et al., (2002), com frutos de acerola.

Apesar dos valores altos de SS, em comparação com os de outras frutíferas do grupo das pequenas frutas, como o morango e a amora-preta, o teor de SS encontrado em fisális, cultivados em Minas Gerais, mostrou-se satisfatório, ao longo da colheita, com valor médio superior a $14^{\circ}$ Brix, citado também pela literatura colombiana, país de maior produção no mundo (Rufato et al., 2008).

Segundo Barros et. al (1996), o alto valor de sólidos solúveis encontrado em frutos pode ser prejudicial para seu tempo de armazenamento, pois, uma alta taxa de açúcares pode estar associada ao aumento da velocidade de deterioração do fruto e da velocidade de fermentação.

$\mathrm{O}$ alto teor de sólidos solúveis encontrado pode ser explicado pela concentração desses compostos, em função da perda de peso (perda de água pelo processo de respiração), concentrando-se mais os açúcares nos tecidos, até 14 dias de armazenagem (Kluge et al., 1995). Pas-

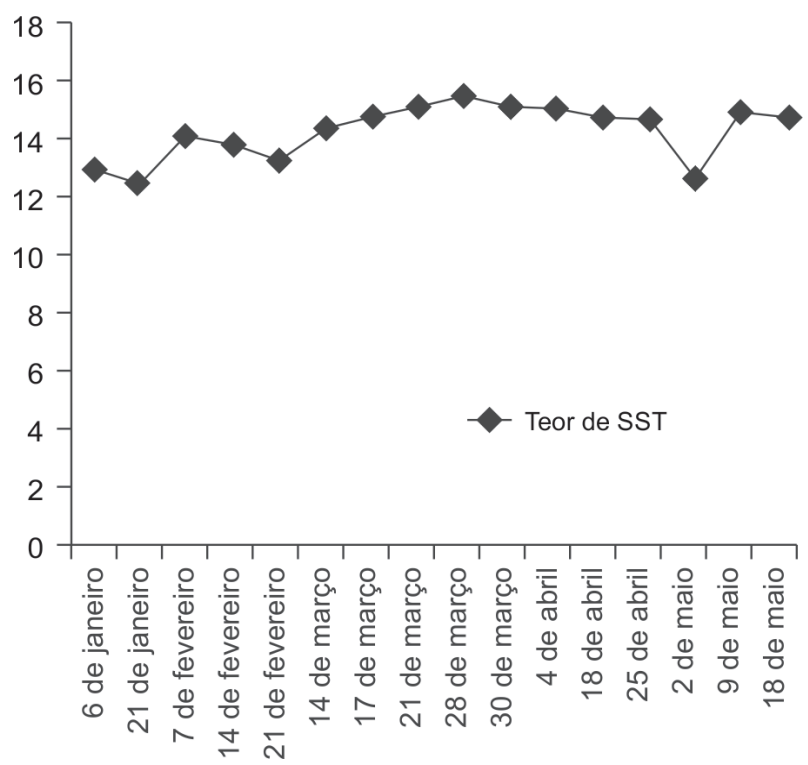

Figura 1. Comportamento do teor de sólidos solúveis (SS) em frutos de fisális ao longo do período de colheita da safra 2010/ 2011. EPAMIG. Maria da Fé, MG. 2012. 
sado esse período, inicia-se a degradação desses compostos que são utilizados no processo de respiração voltando a apresentar valores mais baixos, a partir do $21^{\circ}$. dia (Brackmann et al., 2010).

Os valores de $\mathrm{pH}$ obtidos nos diferentes tratamentos apresentaram resultados significativos, ou seja, diferiram entre si, estatisticamente, em função do tempo de armazenagem. Até o $14^{\circ}$ dia, houve pouca alteração dos valores, que apresentaram média geral de 3,85, no momento da instalação do experimento, e valor de 3,76 , no $14^{\circ}$ dia, decaindo significativamente após essa data, atingindo menores valores para $\mathrm{pH}$ aos 21 e 28 dias após a colheita (Tabela 1). Este resultado pode ser atribuído ao baixo consumo dos ácidos orgânicos como fonte de energia, durante o processo de maturação (Lima et al., 2003).

Juntamente com o acúmulo de ácidos orgânicos, os valores encontrados para a ATT apresentaram-se bastante altos durante todo processo de armazenagem, oscilando entre 14,37 e 15,75. Embora altos, os valores mantiveram-se constantes, não havendo diferenças significativas entre eles.

Negreiros et al. (2008) ressaltaram em seus estudos com maracujazeiro-amarelo a importância da acidez do fruto para a indústria, pois ela desfavorece a manifestação de micro-organismos e, consequentemente, confere maior tempo de conservação do produto. Por outro lado, a acidez proporciona um sabor não muito doce, o que não agrada aos consumidores de frutas in natura.

Resultados semelhantes foram encontrados por Corrêa et al. (2000), em estudos com "fruta-de-lobo" (Solanum lycocarpum St. Hil.), nas quais também não se notou variações significativas para ATT, embora os valores de $\mathrm{pH}$ tenham diminuído ao longo do processo de maturação dos frutos. Os resultados também confirmam a conclusão de Chitarra \& Chitarra (1990), que dizem que frutos com o amadurecimento perdem rapidamente a acidez, mas, em alguns casos, há um pequeno aumento dos teores com o avanço do amadurecimento.

Embora SS e ATT sejam parâmetros avaliados isoladamente, ambos devem ser analisados em conjunto, pois o sabor dos frutos é avaliado pela relação SS/ATT e deve- se ao balanço de ácidos e açúcares (Chitarra \& Chitarra, 2005). Nascimento et al. (2003) citam que o teor de açúcar e a acidez dos frutos podem sofrer variação em decorrência de fatores ambientais, práticas de cultivo, qualidade de luz solar, temperatura, tipo e dosagens de fertilizantes, portanto, com reflexos diretos na relação SS/ATT.

Sendo assim, o resultado encontrado na relação SS/ ATT para frutíferas comumente aumenta durante o processo de maturação, pois os SS tendem a se concentrar, com a perda de água pelo processo de respiração, e a ATT tende a baixar, com a diminuição do teor de ácidos orgânicos, pela ação enzimática. Corroborando os resultados de Amézquita et al. (2008), em seu trabalho com aplicação de cálcio e giberelinas, visando a ampliar a duração pós-colheita de fisális, os resultados obtidos neste experimento evidenciaram valores levemente inferiores para frutos de fisális cultivados no sul de Minas, da ordem de 0,74 a 0,86 , durante os 28 dias de armazenamento, não havendo diferenças significativas entre os períodos de armazenagem avaliados (Tabela 1). Os valores encontrados por Amézquita et al. (2008) foram iguais a 0,8 e 0,97 encontrados em seu tratamento testemunha e no tratamento com maior eficiência, verificado em seu trabalho, respectivamente.

Os teores de vitamina $\mathrm{C}$ encontrados diferiram entre si, decrescendo em função do tempo de armazenamento. Os valores médios iniciais encontrados foram $23,88 \mathrm{mg}$ $100 \mathrm{~g}^{-1}$ de ácido ascórbico de suco, chegando, aos 28 dias, aos valores médios de $6,66 \mathrm{mg} 100 \mathrm{~g}^{-1} \mathrm{de}$ ácido ascórbico de suco (Tabela 1). O teor de vitamina $\mathrm{C}$ tende a diminuir, na maioria dos frutos, durante o processo de maturação, segundo Butt (1980), em função da atuação da enzima denominada ácido ascórbico oxidase, que apresenta maior atividade enzimática em frutos maduros.

Muitos autores escreveram a respeito do decréscimo do teor de vitamina $\mathrm{C}$, ao longo do tempo de armazenamento, em diferentes frutas (Silva et al. (1999), trabalhando com maracujá doce (Passiflora alata Dryander), e Antunes et al. (2003), trabalhando com amora-preta (Rubus sp.).

Gutiérrez et al. (2007), encontraram em amostras silvestres de Physalis peruviana, da região andina sul ame-

Tabela 1. Características físico-químicos de frutos de fisális cultivados em Maria da Fé, sul de Minas Gerais

\begin{tabular}{lccccc}
\hline $\begin{array}{l}\text { Tempo de } \\
\text { armazenagem }\end{array}$ & $\mathbf{S ~ S}$ & $\mathbf{p H}$ & ATT & SS/ATT & $\begin{array}{c}\text { Teor de } \\
\text { ácido ascórbico }\end{array}$ \\
\hline 0 dias & $11,267 \mathrm{~b}^{*}$ & $3,850 \mathrm{a}$ & $15,018 \mathrm{a}$ & $0,750 \mathrm{a}$ & $25,555 \mathrm{a}$ \\
7 dias & $11,666 \mathrm{~b}$ & $3,886 \mathrm{a}$ & $15,756 \mathrm{a}$ & $0,740 \mathrm{a}$ & $23,888 \mathrm{a}$ \\
14 dias & $12,733 \mathrm{a}$ & $3,763 \mathrm{~b}$ & $15,018 \mathrm{a}$ & $0,854 \mathrm{a}$ & $10,555 \mathrm{~b}$ \\
21 dias & $12,400 \mathrm{a}$ & $3,673 \mathrm{~b}$ & $14,378 \mathrm{a}$ & $0,866 \mathrm{a}$ & $8,333 \mathrm{~b}$ \\
28 dias & $12,600 \mathrm{a}$ & $3,673 \mathrm{~b}$ & $15,573 \mathrm{a}$ & $0,818 \mathrm{a}$ & $6,666 \mathrm{~b}$ \\
\hline $\mathrm{CV}$ & 3,81 & 1,46 & 7,54 & 10,80 & 15,68 \\
\hline
\end{tabular}

*Médias seguidas de mesma letra na vertical não diferem estatisticamente, entre si, pelo teste de Scott-Knott, a 5\% de probabilidade. 
ricana, teores de ácido ascórbico que variaram entre 20 e

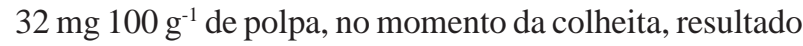
bastante semelhante ao encontrado para os fisális cultivados em Minas Gerais. Também Carrasco \& Zelada (2008) destacam o alto teor de vitamina $\mathrm{C}$ encontrado em $P$. peruviana, em seu estudo com frutas nativas peruanas, com valores equivalentes a 43,3 mg $100 \mathrm{~g}^{-1}$ de ácido ascórbico de polpa.

A avaliação física de frutos é importante, pois frutos com boa aparência e medidas consideradas elevadas são o principal atrativo para o consumidor. Nos frutos de fisális estudados, verificou-se tamanho médio dos frutos maduros próximo de 1,84 e 1,85 centímetros, para diâmetro transversal e diâmetro longitudinal, respectivamente (Figura 2). Estudos realizados por Valência (1985) mostraram diâmetros de $1,6 \mathrm{~cm}$, para frutos de fisális tipo silvestre. Resultados semelhantes ao deste trabalho foram encontrados por Mazorra et al. (2006), que descrevem frutos de fisális ecotipo Colômbia, com diâmetro longitudinal médio de $1,9 \mathrm{~cm}$ e diâmetro transversal de $1,7 \mathrm{~cm}$, atribuindo a superioridade deste ecotipo, em relação ao silvestre, às condições ótimas de cultivo e aos processos de seleção contínua dos melhores frutos, que têm sido realizados pelos produtores daquele país.

No Brasil, estudos realizados por Lima et al. (2009b) no Rio Grande do Sul, analisando variáveis físico-químicas de fisális, em diferentes colorações do cálice, confirmam os resultados obtidos em Minas Gerais, com valores de $1,41 \mathrm{~cm}$, para frutos verdes, a $1,80 \mathrm{~cm}$, para frutos amarelo-arredondados, condição em que o fruto encontra-se completamente maduro.

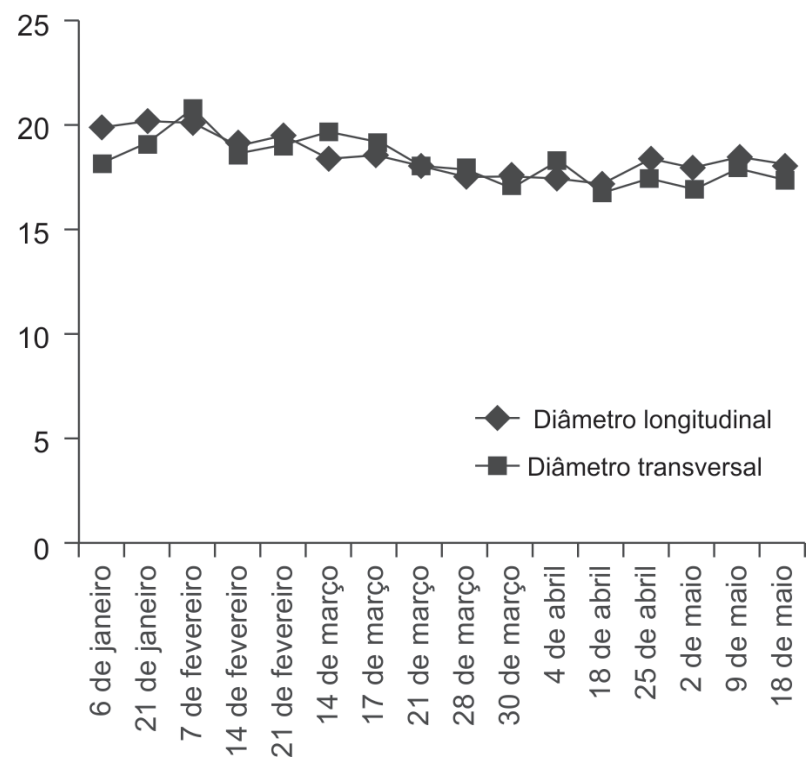

Figura 2. Diâmetro médio de frutos de fisális ao longo do período de colheita na safra 2010/2011. EPAMIG. Maria da Fé, MG. 2012.
A conservação pós-colheita de fisális é, sem dúvida, um fator de grande importância, que está associado a diversos outros elementos. Segundo Chitarra \& Chitarra (1990), são inúmeros os fatores pré e pós-colheita que podem influenciar no tempo de vida de prateleira de frutos e hortaliças. Dentre esses fatores, que podem alongar ou reduzir o tempo de duração de fisális, está a permanência do cálice concrescido, característico do gênero. Durante o armazenamento, a durabilidade da fruta aumenta ainda mais com a presença do cálice, que é muito perecível em temperatura ambiente, especialmente na ausência de seu envoltório natural (Rufato et al., 2008).

Apesar de apresentar aspecto importante na conservação pós-colheita de fisális, o cálice do fruto pode atuar como meio de disseminação de fungos, sendo, portanto, necessária a colheita em estádio avançado de maturação, quando o cálice encontra-se bastante desidratado, ou a utilização de meios artificiais de secagem do cálice (Pareja et al., 2002). Uma vez sendo vantajosa a comercialização da fruta com o cálice, é importante que este não represente massa expressiva, sendo interessantes frutos em que a massa do cálice represente a menor percentagem em relação ao peso total do fruto.

Os frutos estudados apresentaram peso médio, ao longo do período produtivo, de 6,11 g, para frutos sem o cálice, e de 6,75 g, para frutos inteiros. Com os resultados obtidos, individualmente, por colheita, pode-se observar a existência de um pico na produção de frutos com maior peso, concentrado entre o início de fevereiro e meados de março. A partir desta data ocorreu diminuição gradual do peso dos frutos (Figura 3). Esses resultados foram inferiores aos encontrados por Lima et al. (2009), cujos valores variaram de 8,85 a 10,22 g, para diferentes colorações de cálice estudadas.

Ainda analisando-se o peso final dos frutos, pode-se observar que o peso do cálice representou $8,12 \%$ do peso total do fruto, taxa baixa, que estimula a comercialização de fisális com o cálice (Figura 4). O baixo peso do cálice, em relação ao do fruto, no momento da colheita, também foi verificado por Lima et al. (2009b), que o justificaram pela grande quantidade de carboidratos do cálice fornecida ao fruto durante os primeiros 20 dias de desenvolvimento, além da maior capacidade de retenção de água e conteúdo de clorofilas, na fase inicial, quando o fruto apresenta-se verde.

Quanto à perda de massa ao longo do período de conservação, frutos de fisális apresentaram perda média de 3,41 gramas, até os 28 dias de armazenamento, correspondendo a 13,58\% do peso médio inicial dos frutos (Figura 4). Os tratamentos observados não apresentaram diferenças significativas entre si, exceto no armazenamento por 28 dias, quando a queda de peso apresentou-se elevada, diferindo da dos demais tratamentos. As perdas de massa

Rev. Ceres, Viçosa, v. 60, n.6, p. 826-832, nov/dez, 2013 
fresca, em frutos armazenados, decorrem da eliminação da água por transpiração - causada pela diferença de pressão de vapor entre o fruto e o ar no ambiente (Sousa et al., 2000) e dos processos metabólicos de respiração. Sendo assim, pequenos frutos, por apresentarem elevadas taxas respiratórias e grande área de superfície por unidade de volume, perdem bastante água e metabólitos no processo de respiração (Hardenburg et al., 1986).

Perdas de massa elevadas, em outras espécies pertencentes ao grupo das pequenas frutas, foram registradas por diversos autores, como Kluge et al. (1995), em mirtilos, e Antunes et al. (2003), em amora-preta. Estando a perda de massa ligada à desidratação e à perda de metabólitos do

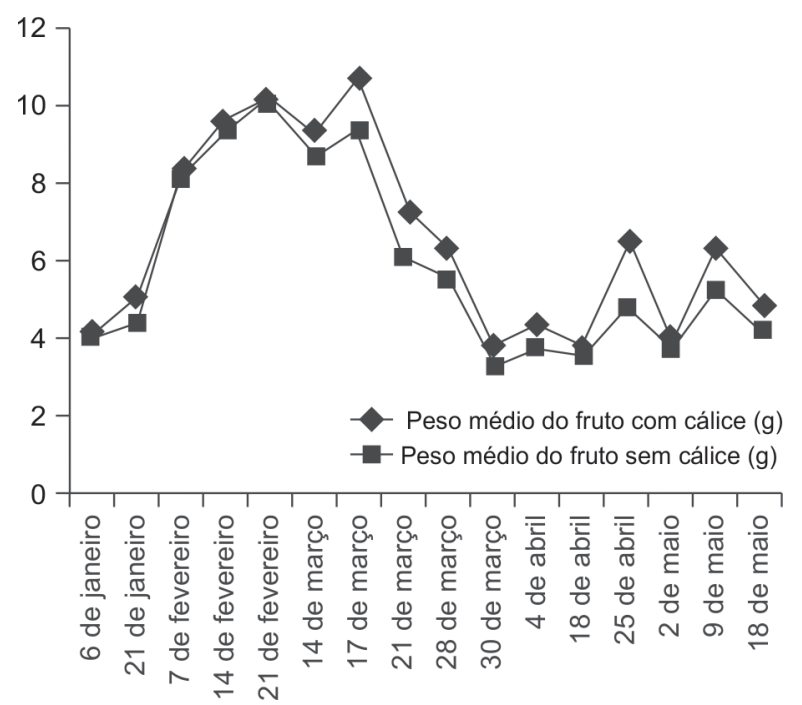

Figura 3. Peso médio de frutos de fisális com e sem o cálice durante o período produtivo da safra 2010/2011. EPAMIG. Maria da Fé/MG. 2012.

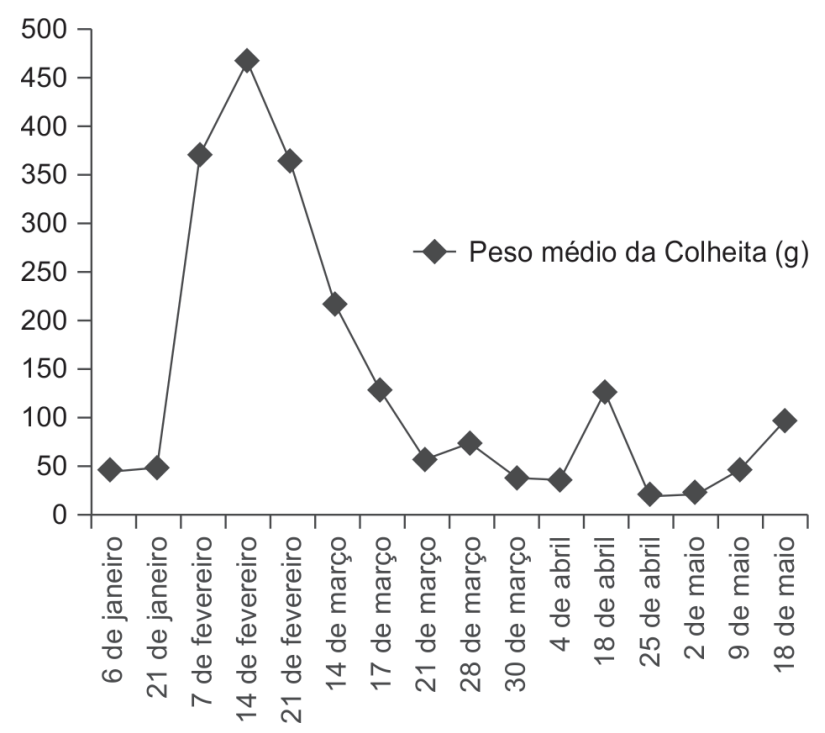

Figura 4. Peso médio por colheita de fisális ao longo do período produtivo na safra 2010/2011. EPAMIG. Maria da Fé, MG. 2012. fruto, outro fator limitante para a comercialização, principalmente de frutos destinados à indústria de sucos e geleias, é o teor de suco do fruto. Os experimentos conduzidos evidenciaram, em fisális, um teor de suco, médio, de $68,30 \%$ não apresentando resultados significativos durante as cinco avaliações, ocorridas no período de 28 dias de armazenamento, embora tenha sido possível verificar uma variação de 62,79 a 74,31\%. Outros frutos, avaliados em condições semelhantes, apresentaram valores altos de rendimento em suco, como os diferentes acessos de acerolas, testadas por Brunini et al. (2004), que atingiram valores entre 50,70 e $76,95 \%$, e amoras-pretas, chegando a $84,00 \%$.

Vários fatores, como espaçamento de plantio, sistema de tutoramento, topografia do terreno, umidade, aeração e forma de tratos culturais empregados, influenciam na densidade de plantas por hectare (Rufato et al., 2008), alterando diretamente o volume de produção por unidade de área de fisális. No experimento montado na Fazenda Experimental de Maria da Fé, a produtividade média por planta foi de 621 gramas, o que totaliza uma produtividade de 1,8 $\mathrm{tha}^{-1}$. A baixa produtividade pode ser atribuída ao pouco conhecimento prático da cultura e ao grande espaçamento utilizado no plantio, reduzindo o número de plantas por hectare.

Segundo dados disponibilizados pela Corporacion Colombia Internacional (CCI, 2002), a produtividade média, na Colômbia, varia entre 14 e $20 \mathrm{t} \mathrm{ha}^{-1}$, em pomares muito bem manejados, com plantios adensados e em regiões que oferecem condições ótimas para desenvolvimento e produção da espécie.

Lima et al. (2010), trabalhando com fisális no Estado do Rio Grande do Sul, encontraram valores médios de $8,54 \mathrm{t} \mathrm{ha}^{-1}$, variando muito, porém, entre diferentes sistemas de tutoramento e épocas de transplantio, confirmando a afirmação de que a produtividade de fisális pode ser facilmente influenciada por diversos fatores, entre eles os tratos culturais. As maiores produtividades de fisális foram observadas nos meses de fevereiro e março (meados), bem como os melhores pesos por fruto.

O cultivo de fisális, na região sul de Minas Gerais, apresentou resultados positivos, com valores que podem ser comparados aos das regiões tradicionais no cultivo da fruta, o que pode oferecer ao produtor condições básicas para concorrer no mercado de frutos importados, podendo oferecer um produto de qualidade semelhante, com preços menores. Levando-se em consideração o total desconhecimento a respeito do comportamento fisiológico, fitossanitário e fitotécnico de Physalis peruviana, para as condições brasileiras, os resultados iniciais projetam a fruta como promissora para a região, podendo oferecer ao pequeno produtor uma nova alternativa de renda. Contudo, mais estudos fazem-se necessários para o estabelecimento definitivo da cultura. 


\section{CONCLUSÕES}

O teor de vitamina $\mathrm{C}$ encontrado em fisális apresentou-se elevado, acima do de muitas frutíferas comumente conhecidas por essas características.

Frutos de fisális podem ser armazenados por 28 dias, a $5^{\circ} \mathrm{C}$.

Maiores produtividades por colheita e frutos mais pesados foram verificados nos meses de fevereiro e metade de março.

\section{REFERÊNCIAS}

Amézquita N, Balaguera-López HEJG \& Álvarez-Herrera JG (2008) Efecto de la aplicación precosecha de giberelinas y cálcio en la producción, calidad y rajado del fruto de uchuva (Physalis peruviana L.). Revista Colombiana de Ciencias Hortícolas, 2:133-144.

Antunes LEC, Duarte Filho J \& Souza CM (2003) Conservação pós-colheita de frutos de amoreira-preta. Pesquisa Agropecuária Brasileira, 38:413-419.

ALMG- Assembléia Legislativa do estado de Minas Gerais (2008) Banco de dados. Belo Horizonte, 2005. Disponível em: <http:/ /www.almg.gov.br/index.asp?grupo=estado\&diretorio $=$ munmg \&arquivo $=$ municipios $>$. Acessado em: 05 de novembro de 2012 .

Barros RS, Finger FL \& Magalhães MM (1996) Changes in nonstructural carbohydrates in developing fruit of Myrciaria jabuticaba. Scientia Horticulturae, 16:209-215.

Benassi MT \& Antunes AJA (1988) Comparison of metaphosphoric and oxalic acids as extractant solutions for the determination of vitamin $\mathrm{C}$ in selected vegetables. Arquivos de Biologia e Tecnologia, 31:507-513.

Brackmann A, Weber A, Giehl RFH, Einsermann AC, Sautter CK, Gonçalves ED \& Antunes LEC (2010) Armazenamento de mirtilo 'Bluegem' em atmosfera controlada e refrigerada com absorção e inibição do etileno. Revista Ceres, 57:06-11.

Brunini MA, Macedo NB, Coelho CV \& Siqueira GF (2004) Caracterização física e química de acerolas provenientes de diferentes regiões de cultivo. Revista Brasileira de Fruticultura, 26:486-489.

Butt VS (1980) Direct oxidases and related enzymes. In: Stumpf PK \& Conn EE (Eds.) The biochemistry of plants: a comprehensive treatise. New York, Academic. 2:81-123.

Carrasco RR \& Zelada CRE (2008) Determinación de la capacidad antioxidante y compuestos bioactivos de frutas nativas peruanas. Revista de La Sociedad Quimica del Perú, 74:108-124.

Castañeda R (1961) Frutas Silvestres de Colombia. Bogota, Editora San Juan Eudes. 342p.

Chitarra MIF \& Chitarra AB (1990) Pós-colheita de frutos e hortaliças: fisiologia e manuseio. Lavras, ESAL/FAEPE. 320p.

Chitarra MIF \& Chitarra AB (2005) Pós-colheita de frutos e hortaliças: Fisiologia e Manuseio. 2 ed. Lavras, UFLA. 785p.

CCI - Corporacion Colombia Internacional (2002) Uchuva. Perfil de producto. Revista Inteligência de Mercados, 13:1-12.

Corrêa AD, Abreu CMP, Santos CD \& Ribeiro LJ (2000) Determinação de alguns constituintes químicos de interesse nutricional da fruta-de-lobo (Solanum lycocarpum St. Hil.). Ciências Agrotécnicas, 24:130-135.

Ferreira DF (2000) Sistema de análises de variância para dados balanceados. Versão 4.1. Lavras, UFLA. (Software estatístico).
Gutiérrez TM, Hoyos OL \& Páez MI (2007) Determinación del contenido de ácido ascórbico en uchuva (Physalis peruviana l.), por cromatografia líquida de alta resolución (CLAR). Biotecnología en el Sector Agropecuario y Agroindustrial, 5:70-79.

Hardenburg RE, Watada AE \& Wang CY (1986) The commercial storage of fruits, vegetables, and florist, and nursery stocks. Washington, USDA: Agricultural Research Service.130p.

Instituto Adolfo Lutz (1985) Normas analíticas, métodos químicos e físicos para análise de alimentos. v.1. São Paulo. 371p.

Kluge RA, Hoffmann A, Nachtigal JC, Bilhalva AB, Santos AM (1995) Frigoconservação de frutos de mirtilo (Vaccinium ashei Reade) cv. Climax. Revista Brasileira de Agrociência, 1:185188 .

Lima CSM, Gonçalves MA, Tomaz ZFP, Rufato AR \& Fachinello JC (2010) Sistemas de tutoramento e épocas de transplante de physalis. Ciência Rural, 40:2472-2479.

Lima CSM (2009a) Fenologia, sistemas de tutoramento e produção de Physalis peruviana na região de Pelotas, RS. Dissertação de Mestrado. Universidade Federal de Pelotas, Pelotas. 117p.

Lima CSM, Severo J, Manica-Berto R, Silva JA, Rufato L \& Rufato AR (2009b) Características físico-químicas de Physalis em diferentes colorações do cálice e sistemas de condução. Revista Brasileira de Fruticultura, 31:1060-1068.

Lima MAC, Alves RE, Filgueiras HAC \& Enéas-Filho J (2003) Respiratory behavior and postharvest quality of 'Morada' soursop (Annona muricata L.) at room temperature. Revista Brasileira de Fruticultura, 25:49-52.

Mazorra MF, Quintana AP, Miranda D, Fischer G \& Valencia MC (2006) Aspectos anatómicos de la formación y crecimiento del fruto de uchuva Physalis peruviana (Solanaceae). Acta Biológica Colombiana, 11:69-81.

Nascimento WMO, Tomé AT, Oliveira, MSP, Müller CH, Carvalho JEU (2003) Seleção de progênies de maracujazeiro-amarelo (Passiflora edulis f. flavicarpa) quanto à qualidade de frutos. Revista Brasileira de Fruticultura, 25:186-188.

Negreiros JRS, Araújo Neto SE, Álvares VS, Lima VA \& Oliveira TK (2008) Caracterização de frutos de progênies de meiosirmãos de maracujazeiro-amarelo em Rio Branco - Acre. Revista Brasileira de Fruticultura, 30:431-437.

Nogueira RJMC, Moraes JAPV, Burity HA \& Silva Júnior JF (2002) Efeito do estádio de maturação dos frutos nas características físico-químicas de acerola. Pesquisa Agropecuária Brasileira, $37: 463-470$.

Pareja JLZ, Cardona AS, Londoñon M \& Díaz C (2002) Manejo del cultivo de La uchuva en Colombia, boletín técnico. Corporación Colombiana de Investigación Agropecuaria Corpoica, regional 4, Centro de Investigación La Selva, 42p. (Boletim técnico).

Pereira B (2007) Frutas finas. Revista Frutas e Derivados, 2:14-18.

Rufato L, Rufato AR, Schlemper C, Lima CSM \& Kretzschmar AA (2008) Aspectos técnicos da cultura da Physalis. Pelotas, UFpel. 101p.

Silva AP, Domingues MCS, Vieites RL \& Rodrigues JD (1999) Fitorreguladores na conservação pós-colheita do maracujá doce (Passiflora alata dryander) armazenado sob refrigeração. Ciência e Agrotecnologia, 23:643-649.

Sousa RF, Filgueiras HAC, Costa JTA, Alves RE \& Oliveira AC (2000) Armazenamento de ciriguela (Spondia purpurea L.) sob atmosfera modificada e refrigeração. Revista Brasileira de Fruticultura, 22:334-338.

Valência M (1985) Anatomía del fruto de uchuva (Physalis peruviana L.). Acta Biológica Colombiana, 1:63-89. 